\title{
EDITORIAL
}

\section{Challenges in deep brain stimulation for depression} Albert Fenoy (iD

The University of Texas Health Science Center at Houston, Houston, TX, USA.

Treatment-resistant depression (TRD) is an incapacitating condition affecting millions worldwide. Many of these patients have increased healthcare utilization but remain sick, justifying the urgent need to develop and implement improved strategies, such as deep brain stimulation (DBS).

The various studies of DBS targets for TRD over the last $10+$ years have shown variable response rates; the subgenual cingulate (Cg25), the most popular target, only yielded a combined response rate of near $50 \%$ at 12 months ${ }^{1}$ from a pooled 77 patients at eight centers. Other targets shared the same problem, where stimulation parameters have been blindly adjusted across variable anatomic locations to incur acute mood changes rather than be titrated to a defined objective measurement, leading to overall mediocre response rates.

We need to 1) structurally, 2) functionally, and 3) electrophysiologically define each individual's dysfunctional affective circuit and use these definitions to help identify biomarkers of stimulation response that can optimize the target.

Structurally, we are getting closer to finding a DBS target for reproducible, significant results. Within a given target, variability in response to DBS can be due to variability of electrode position: millimeter differences within the same target in different patients means that a slightly different subset of subcortical fibers is modulated. Structural tractography - deterministic in surgical planning, probabilistic for population analysis - is a key tool to help make targeting results reproducible. This is now common across all centers, and is a large reason why a recent cohort of $\mathrm{Cg} 25$ patients had an $82 \%$ response rate at 1 year. ${ }^{1}$ The use of tractography has elucidated fiber pathways such as the medial forebrain bundle (MFB), which when stimulated has led to significantly rapid responses of over $80 \%$ before 6 months. ${ }^{2,3}$ This branch of the MFB represents the outflow fibers from the ventral tegmental area, an important node within the reward pathway, which is also part of a larger affective circuit connecting Cg25 and other DBS depression targets to certain prefrontal cortical mediators, dysfunction of which is known to underlie depression.

Functionally, there are differences between targets which lead to anti-depressive effect. Each DBS white-matter target can differentially modulate this aberrantly wired network,

Correspondence: Albert Fenoy, 6400 Fannin, Suite 2800, 77225, Houston, TX, USA.

E-mail: Albert.J.Fenoy@uth.tmc.edu

Submitted Jan 28 2020, accepted Feb 04 2020, Epub Apr 302020. with possibly greater acuity at specific nodes due to more concentrated fibers, which could be argued to lead to a faster anti-depressive effect. The problem is how to measure anti-depressant effect accurately: daily, weekly, monthly, by reaction to new stressors, etc., and not just by a subjective questionnaire that leads to a score that can be tracked over time. There should be some or multiple other objective measures, such as observation of improved demeanor, arousal, appetitive motivation, interpersonal relationships, or - even better - an actual biomarker, a signal that the circuit has been positively altered and success has been found.

This is where functional imaging comes into the picture. We need to have some objective measure of success - a biomarker. So far, results are elusive, but we are getting closer. Metabolic changes visualized on ${ }^{18} \mathrm{~F}$-fluorodeoxyglucose positron emission tomography (FDG-PET) were thought to be an answer, but these changes are not seen in all depressed patients and are not unique to DBS. Perhaps connectivity seen with resting-state functional MRI (fMRI) of the default mode network and changes therein after anti-depressive stimulation can be used as a guide, but we are just beginning to sort through this analysis.

Even though these targets all lie along the same circuit, they are obviously not the same, with differential effects. More importantly, to complicate matters, is the large problem that not all depression is the same. Previous clinical trials have tried to stratify the most treatment resistant, but even so there are different phenotypes that seem more likely to have success with a given target, owing to the specific fiber tracts modulated. Potentially, we will be able to use functional imaging as a biomarker to select those specific patients (e.g., anhedonic, melancholic) that can be remedied by a specific target known to have better effect for that depressive subtype.

Depression is a chronic, debilitating illness, secondary to years of aberrant wiring of an affective circuit. Stimulation to redirect fiber modulation and recruit certain areas of the cortex can have effects that are acute, but we need more proof that a change actually occurs.

From an electrophysiological standpoint, proving connectivity to certain cortical mediators may help optimize outcome. There is mounting evidence that modulation of 
the prefrontal cortex (PFC), specifically the ventral PFC, is involved with regulation of mood. Functional imaging, tractography modeling, ${ }^{1,2}$ and electrophysiology ${ }^{4,5}$ responses to stimulation all point to specific cortical regions that appear to be very pertinent to mood. Obviously, differences in subcortical targets differentially influence these cortical regions, leading to differential effects.

If we can measure theta oscillations representative of effortful cognitive control and how they might be positively modulated by DBS, ${ }^{4}$ or else record theta band changes congruent with acute mood change, ${ }^{5}$ this would serve as an objective measure to gauge stimulation effect: to know if a sufficient stimulus threshold has been attained; to know if the target is effective and then identify which target is most strategically poised to modulate this circuit; and to know if a true change has been incurred instead of being held hostage to remedying the subjective daily mood swings of chronically ill patients.

Much more work needs to be done on this frontier, both with intracranial and noninvasive recordings, to understand how this complex affective network is modulated by stimulation. Together, with both structural modeling and functional imaging, the use of electrophysiological correlates to identify objective effects can help direct us toward our ultimate goal of finding a suitable DBS target for depression.

\section{Disclosure}

The author is a consultant for Medtronic, Inc.

\section{References}

1 Riva-Posse P, Choi KS, Holtzheimer PE, Crowell AL, Garlow SJ, Rajendra JK, et al. A connectomic approach for subcallosal cingulate deep brain stimulation surgery: prospective targeting in treatmentresistant depression. Mol Psychiatry. 2018;23:843-9.

2 Fenoy AJ, Schulz PE, Selvaraj S, Burrows CL, Zunta-Soares G, Durkin $\mathrm{K}$, et al. A longitudinal study on deep brain stimulation of the medial forebrain bundle for treatment-resistant depression. Transl Psychiatry. 2018;8:111.

3 Schlaepfer TE, Bewernick BH, Kayser S, Mädler B, Coenen VA. Rapid effects of deep brain stimulation for treatment-resistant major depression. Biol Psychiatry. 2013;73:1204-12.

4 Widge AS, Zorowitz S, Basu I, Paulk AC, Cash SS, Eskandar EN, et al. Deep Brain stimulation of the internal capsule enhances human cognitive control and prefrontal cortex function. Nat Commun. 2019;10:1536.

5 Rao VR, Sellers KK, Wallace DL, Lee MB, Bijanzadeh M, Sani OG, et al. Direct electrical stimulation of lateral orbitofrontal cortex acutely improves mood in individuals with symptoms of depression. Curr Biol. 2018;28:3893-902.e4. 